\title{
On the Mixed Discretization of the Time Domain Magnetic Field Integral Equation
}

\author{
H. A. Ülkü ${ }^{1} \quad$ I. Bogaert ${ }^{2} \quad$ K. Cools ${ }^{3} \quad$ F. P. Andriulli ${ }^{4} \quad$ H. Bağc1 ${ }^{1}$
}

Abstract - Time domain magnetic field integral equation (MFIE) is discretized using divergenceconforming Rao-Wilton-Glisson (RWG) and curl-conforming Buffa-Christiansen (BC) functions as spatial basis and testing functions, respectively. The resulting mixed discretization scheme, unlike the classical scheme which uses RWG functions as both basis and testing functions, is "proper": Testing functions belong to dual space of the basis functions. Numerical results demonstrate that the marching on-in-time (MOT) solution of the mixed discretized MFIE yields more accurate results than that of classically discretized MFIE.

\section{INTRODUCTION}

Magnetic field integral equation (MFIE) is a second kind integral equation, i.e., it is constructed as a summation of an identity and a linear operator. Because of the identity operator, the domain and range of the MFIE operator are identical and a consistent discretization scheme should use basis and testing functions, which belong to dual spaces of each other [1]. In particular, the testing function should be in the dual space of the MFIE operator's range (and domain) to obtain a "proper" discretization scheme. Classical marching on-intime (MOT) based MFIE solvers expand the unknown surface current density using divergence-conforming Rao-Wilton-Glisson (RWG) functions [2] in space and polynomial functions in time. To obtain a proper discretization, spatial testing should be done using curl-conforming $\hat{\mathbf{n}} \times \mathrm{RWG}$ functions, which belong to dual space of the divergence conforming RWG basis functions. However, resulting MOT matrix becomes singular and cannot be inverted accurately at every time step as required by the MOT scheme. Therefore, classical implementations use RWG functions (but not their duals) for spatial testing and violates the requirement of the proper discretization described above. Even though the solution of the MOT matrix system resulting from this discretization scheme converges fast, it yields inaccurate results.

In this work, time domain MFIE is discretized using the mixed discretization scheme, which is originally proposed for discretizing the frequency domain MFIE [3]. Mixed discretization scheme makes use of recently proposed Buffa-Christiansen (BC) functions [4]-[5] to produce well-conditioned MOT matrices without violating the requirement of the proper discretization described above. Current density is expanded using divergence-conforming RWG functions in space and spatial testing is carried out using curl-conforming $\hat{\mathbf{n}} \times \mathrm{BC}$ functions, which belong to dual space of the divergence-conforming RWG functions.

\footnotetext{
${ }^{1}$ Division of Physical Science and Engineering, King Abdullah University of Science and Technology, Thuwal, KSA e-mail: huseyin.ulku@kaust. edu.sa, hakan.bagci@kaust. edu. sa tel.: +9662 8084362, fax: +96628080140.

${ }^{2}$ Department of Information Technology, Ghent University, Ghent, Belgium.

e-mail: Ignace.Bogaerteintec.ugent.be

${ }^{3}$ Department of Electrical and Electronic Engineering, University of Nottingham, Nottingham, UK.

e-mail: Kristof.Cools@nottingham.ac.uk

${ }^{4}$ Microwave Department, TELECOM Bretagne, Brest, France.

e-mail: francesco.andriullietelecom-bretagne.eu
} 
Numerical results demonstrate that the MOT solution of the mixed discretized MFIE yields more accurate results than that of the classically discretized MFIE, as expected.

\section{MAGNETIC FIELD INTEGRAL EQUATION}

Time domain MFIE, which is obtained by enforcing the magnetic field boundary condition on perfect electrically conductor (PEC) scatterer surfaces, reads

$$
\begin{aligned}
& \hat{\mathbf{n}}(\mathbf{r}) \times \mathbf{H}^{\mathrm{inc}}(\mathbf{r}, t)=\frac{1}{2} \mathbf{J}(\mathbf{r}, t) \\
& \quad-\hat{\mathbf{n}}(\mathbf{r}) \times \nabla \times \int_{S} \mathbf{J}\left(\mathbf{r}^{\prime}, t\right) * \frac{\delta(t-R / c)}{4 \pi R} d \mathbf{r}^{\prime}
\end{aligned}
$$

Here, $S$ denotes the scatter surface, $\mathbf{H}^{\text {inc }}(\mathbf{r}, t)$ is the (essentially) band-limited incident magnetic field, $\mathbf{J}(\mathbf{r}, t)$ is unknown current density, $R=\left|\mathbf{r}-\mathbf{r}^{\prime}\right|$ is the distance between the source point $\mathbf{r}^{\prime}$ and the observation point $\mathbf{r}, \hat{\mathbf{n}}(\mathbf{r})$ is the outward-pointing unit normal vector defined at $\mathbf{r}, c$ is the speed of light in the medium, where $S$ resides, * denotes the temporal convolution operation, and $\delta($.$) is the Dirac delta function. To$ numerically solve (1) for the unknown current density $\mathbf{J}(\mathbf{r}, t)$, MOT scheme expands $\mathbf{J}(\mathbf{r}, t)$ in terms of temporal and spatial basis functions:

$$
\mathbf{J}(\mathbf{r}, t)=\sum_{n=1}^{N} \sum_{i=1}^{N_{t}} I_{n, i} T_{i}(t) \mathbf{b}_{n}(\mathbf{r})
$$

In (2), $\mathbf{f}_{n}(\mathbf{r})$ denotes the $\mathrm{n}^{\text {th }}$ spatial basis function, which is chosen as the RWG basis function, $T_{i}(t)$ is the $\mathrm{i}^{\text {th }}$ temporal basis function, which is chosen as the shifted polynomial Lagrange interpolation function, and $I_{n, i}$ is the unknown coefficient associated with the $\mathrm{n}^{\text {th }}$ spatial and $\mathrm{i}^{\text {th }}$ temporal basis function. Here, $N$ and $N_{t}$ are numbers of spatial basis functions and time steps, respectively. Substituting (2) in (1) and testing the resulting equation with testing functions $\mathbf{t}_{m}(\mathbf{r}), m=1, . ., N$, in space and $\delta\left(t-t_{j}\right), j=1, . ., N_{t}$ in time, yield the linear MOT system:

$$
\begin{aligned}
& \int_{S_{m}} \mathbf{t}_{m}(\mathbf{r}) \cdot \hat{\mathbf{n}}(\mathbf{r}) \times \mathbf{H}^{\mathrm{inc}}\left(\mathbf{r}, t_{j}\right) d \mathbf{r}= \\
& \quad \sum_{n=1}^{N} \sum_{i=1}^{j} I_{n, i}\left\{\frac{1}{2} \int_{S_{m}} \mathbf{t}_{m}(\mathbf{r}) \cdot T_{i}\left(t_{j}\right) \mathbf{f}_{n}(\mathbf{r}) d \mathbf{r}-\int_{S_{m}} \mathbf{t}_{m}(\mathbf{r}) \cdot \hat{\mathbf{n}}(\mathbf{r}) \times\left[T_{i}\left(t_{j}\right) * \mathbf{H}_{n}\left(\mathbf{r}, t_{j}\right)\right] d \mathbf{r}\right\} .
\end{aligned}
$$

Here, $t_{j}=j \Delta t$ and $\Delta t$ is the time step size and $\mathbf{H}_{n}(\mathbf{r}, t)$ is the magnetic field due to impulsively excited RWG basis function. It should be noted here that $\mathbf{H}_{n}(\mathbf{r}, t)$ is evaluated analytically as described in [6]-[8]; $\mathbf{H}_{n}(\mathbf{r}, t)$ does not have any spatial singularities, therefore there is no need for a singularity treatment scheme to enhance accuracy of the resulting matrix elements. Additionally, for polynomial $T_{i}(t)$, temporal convolution in (3) can be evaluated in analytically [6]-[8]. 
The choice of testing basis function $\mathbf{t}_{m}(\mathbf{r})$ determines type of the discretization strategy as described next in Section 2.1 and 2.2, and one of factors that determine the accuracy of the solution as demonstrated by the numerical results presented in Section 3.

\subsection{Classical Discretization Strategy}

Classical Galerkin discretization strategy uses divergence-conforming RWG functions for spatial testing, i.e., $\mathbf{t}_{m}(\mathbf{r})=\mathbf{f}_{m}(\mathbf{r})$ in (3), where $\mathbf{f}_{m}(\mathbf{r})$ denotes the $\mathrm{m}^{\text {th }} \mathrm{RWG}$ function. Even though the Gram matrix [first term on the right hand side of (3)] resulting from this type of discretization is well-conditioned, this discretization scheme is not proper: Testing and basis functions belong to the same function space; see Section 1 for a brief explanation and see [3] for more details.

\subsection{Mixed Discretization Strategy}

Mixed discretization strategy uses curl-conforming rotated BC functions for spatial testing, i.e., $\mathbf{t}_{m}(\mathbf{r})=\hat{\mathbf{n}}(\mathbf{r}) \times \mathbf{g}_{m}(\mathbf{r})$ in (3), where $\mathbf{g}_{m}(\mathbf{r})$ denotes the $\mathrm{m}^{\text {th }}$ BC function. After several mathematical manipulations, (3) can be rewritten as

$$
\begin{aligned}
& \int_{S_{m}} \mathbf{g}_{m}(\mathbf{r}) \cdot \mathbf{H}^{\mathrm{inc}}\left(\mathbf{r}, t_{j}\right) d \mathbf{r}= \\
& \sum_{n=1}^{N} \sum_{i=1}^{j} I_{n, i}\left\{\frac{1}{2} \int_{S_{m}}\left[\hat{\mathbf{n}}(\mathbf{r}) \times \mathbf{g}_{m}(\mathbf{r})\right] \cdot T_{i}\left(t_{j}\right) \mathbf{f}_{n}(\mathbf{r}) d \mathbf{r}-\int_{S_{m}} \mathbf{g}_{m}(\mathbf{r}) \cdot \nabla \times\left[T_{i}\left(t_{j}\right) * \mathbf{H}_{n}\left(\mathbf{r}, t_{j}\right)\right] d \mathbf{r}\right\} .
\end{aligned}
$$

It should be noted here that $\hat{\mathbf{n}}(\mathbf{r}) \times \mathbf{g}_{m}(\mathbf{r})$ belong to the dual space of $\mathbf{f}_{n}(\mathbf{r}) . \mathbf{g}_{m}(\mathbf{r})$ are defined on the barycentric refinement of the initial mesh, where $\mathbf{f}_{n}(\mathbf{r})$ are constructed; additionally they are linear combinations of RWG functions constructed on the barycentric mesh [9]-[10]. MOT system (4) can easily be implemented using existing codes that can account for (3) with RWG functions defined on the barycentric mesh.

Mixed discretization scheme satisfies the proper discretization condition (see Section 1 for a brief explanation and see [3] for more details) and generates a more accurate MOT system as shown by the numerical results presented in the next section.

\section{NUMERICAL RESULTS}

To demonstrate that the mixed discretization scheme produces more accurate MOT systems than the classical scheme, transient scattering from a unit sphere residing in free space is investigated. The sphere is discretized with 720 triangular patches. The excitation is chosen as modulated Gaussian plane wave propagating in $-\hat{\mathbf{z}}$ direction:

$$
\mathbf{H}^{i n c}(\mathbf{r}, t)=-\hat{\mathbf{y}} \cos \left[2 \pi f_{0}\left(t-t_{p}+\mathbf{r} \cdot \hat{\mathbf{z}} / c\right)\right] e^{-\frac{\left(t-t_{p}+\mathbf{r} \cdot \hat{\mathbf{z}} / c\right)^{2}}{2 \sigma^{2}}}
$$


where $f_{0}=75 \mathrm{MHz}$ is the modulation frequency, $f_{b w}=30 \mathrm{MHz}$ is the effective bandwidth, $\sigma=7 /\left(2 \pi f_{b w}\right)$ is a measure of pulse duration, and $t_{p}=3.5 \sigma$ is the delay. Time step size is chosen as $\Delta t=0.1 /\left(f_{0}+f_{b w}\right)$. Third order polynomial Lagrange interpolation function is used as $T_{i}(t)$. Both MOT systems in (3) and (4) are solved for $N_{t}=2000$ time steps. Figure 1 plots the coefficients of the 1st RWG basis function, which are obtained by solving (3) and (4). Figure 2 plots the relative norm error in the radar cross section (RCS) with respect to Mie series solution. The error is computed using

$$
\operatorname{err}^{R C S}\left(f_{j}\right)=\sqrt{\frac{\sum_{m=1}^{N_{e}}\left|\sigma_{\text {time }}\left(f_{j}, \theta_{m}, \phi\right)-\sigma_{\text {Mie }}\left(f_{j}, \theta_{m}, \phi\right)\right|^{2}}{\sum_{m=1}^{N_{e}}\left|\sigma_{\text {Mie }}\left(f_{j}, \theta_{m}, \phi\right)\right|^{2}}} .
$$

Here, $N_{\theta}=181, \theta_{m}=(m-1), \phi=0, f_{j}=j 180 \mathrm{kHz}$, and $\sigma_{\text {time }}\left(f_{j}, \theta_{m}, \phi\right)$ and $\sigma_{\text {Mie }}\left(f_{j}, \theta_{m}, \phi\right)$ are RCS samples obtained at these points by Fourier transforming the time-domain MOT results and the Mie series solution, respectively. Figure 2 clearly demonstrates the superior accuracy of the mixed discretization scheme.

\section{References}

[1] P. Ylä-Oijala, S. P. Kiminki, K. Cools, F.P. Andriulli and S. Järvenpää, "Mixed discretization schemes for electromagnetic surface integral equations," International Journal of Numerical Modelling: Electronic Networks, Devices and Fields, 2012 (DOI: 10.1002/jnm.844).

[2] S. M. Rao, D. R. Wilton, and A. W. Glisson, "Electromagnetic scattering by surfaces of arbitrary shape," IEEE Transactions on Antennas and Propagation, vol. 30, no. 3, pp. 408-418, May 1982.

[3] K. Cools, F. P. Andriulli, D. De Zutter, and R. Michielssen, "Accurate and conforming mixed discretization of the MFIE,” IEEE Antennas and Wireless Propagation Letters, vol. 10, pp. 528-531, 2011.

[4] A. Buffa and S. H. Christiansen, "A dual finite element complex on the barycentric refinement," Mathematics of Computation, vol. 260, pp. 1743-1769, 2007.

[5] A. Buffa and S.H. Christiansen, "A dual finite element complex on the barycentric refinement," Tech. Rep. PV-18 IMATI-CNR, 2005.

[6] H. A. Ülkü and A. A. Ergin, "Analytical evaluation of transient magnetic fields due to RWG current bases,” IEEE Transactions on Antennas and Propagation, vol. 55, no. 12, pp. 3565-3575, Dec. 2007.

[7] H. A. Ülkü and A. A. Ergin, "Application of analytical retarded-time potential expressions to the solution of time domain integral equations," IEEE Transactions on Antennas and Propagation, vol. 59, no. 11, pp. 4123-4131, Nov. 2011.

[8] H. A. Ülkü and A. A. Ergin, "Application of analytical expressions of transient potentials to the MOT solution of integral equations," in Proc. IEEE AP-S Int. Symp., San Diego, CA, 2008. 
[9] F. P. Andriulli, K. Cools, H. Bagci, F. Olyslager, A. Buffa, S. Christiansen, “A multiplicative Calderon preconditioner for the electric field integral equation," IEEE Transactions on Antennas and Propagation, vol. 56, no. 8, pp. 2398-2412, Aug. 2008.

[10] H. Bagci, F.P. Andriulli, K. Cools, F. Olyslager, E. Michielssen, "A Calderon multiplicative preconditioner for the combined field integral equation”, IEEE Transactions on Antennas and Propagation, vol. 57, no. 10 , pp. 3387-3392, Oct. 2009.

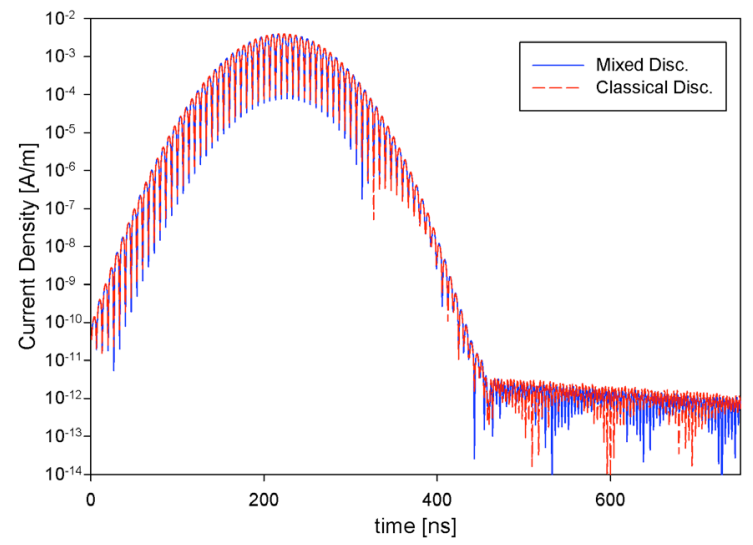

Figure 1: Coefficients of the 1st RWG basis function obtained by solving the MOT systems (3) and (4).

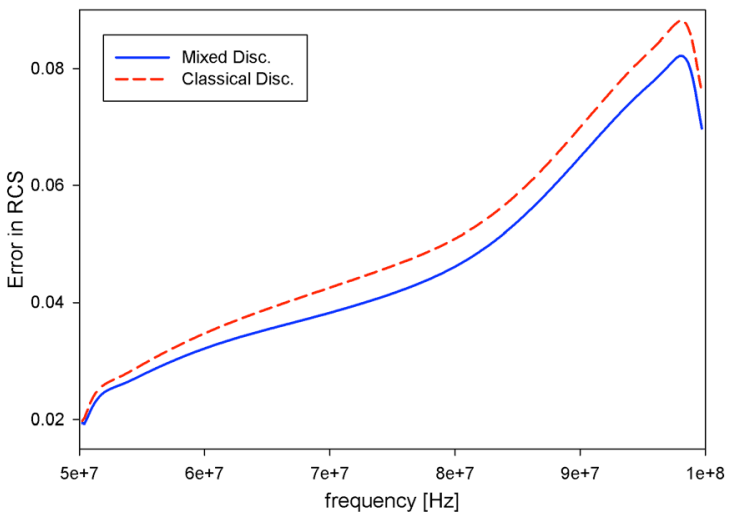

Figure 2: $e r r^{R C S}(f)$ obtained from solutions of the MOT systems in (3) and (4). 